\section{Avaliação da qualidade da atenção básica utilizando a doença respiratória da infância como traçador, em um distrito sanitário do município de São Paulo}

\section{Primary health care quality evaluation using respiratory diseases as tracer in a health district in the city Sao Paulo}

Oswaldo Yoshimi Tanaka 1

Antônio Carlos Gomes do Espírito Santo 2

I Departamento de Prática de Saúde Pública. Faculdade de Saúde Pública. Universidade de São Paulo. São Paulo, SP, Brasil. 2 Programa Integrado de Pós-Graduação em Saúde Coletiva. Departamento de Medicina Social. Centro de Ciências da Saúde. Universidade Federal de Pernambuco. Av. Morais Rego, s. n. Prédio do Centro de Ciências da Saúde. Cidade Universitária Recife, PE, Brasil. CEP: 50.670-901.

E-mail: ag.santo@yahoo.com.br

\begin{abstract}
Objectives: to assess quality of the primary care using respiratory diseases as a tracer.

Methods: the evaluative approach is based on Donabedian's referencial connected to Kessner's tracers methodology. The sample is formed by 768 children from 0 to 5 years old, presenting respiratory symptoms, attended at seven basic health units from the Sanitary District of Brasilandia, in the city of São Paulo, State of São Paulo, Brazil. Medical registrations and interviews with mothers or responsibles were the information sources.

Results: more accurate child medical examination evidences significant association with favorable evolution, mainly on moderate clinical forms. Although aspects related to the accessibility demonstrated by the health team seem to influence decisively on care quality evaluated by users, mainly the medical doctor. Mothers education level appears to have no influence in the favorable evolution.

Conclusions: the tracer methodology has the potentiality to facilitate the critical analysis of the health care. It is important to focus on the outcome of the health care. The children respiratory diseases are important tracer for the quality assessment on health services.
\end{abstract}

Key words Primary health care, Health services evaluation, Evaluation methods

\section{Resumo}

Objetivos: avaliar a qualidade dos serviços de atenção básica utilizando a doença respiratória da infância como traçador.

Métodos: a abordagem avaliativa se baseia no referencial de Donabedian acoplado à metodologia da condição traçadora de Kessner. A amostra é composta de 768 crianças de zero a cinco anos de idade, com queixas respiratórias, atendidas em sete Unidades Básicas de Saúde do Distrito Sanitário de Brasilândia, município de São Paulo, São Paulo, Brasil. As fontes de dados foram prontuários e entrevistas com mães ou responsáveis.

Resultados: o exame médico mais acurado da criança mostrou-se significativamente associado com a evolução favorável dos quadros atendidos. $O$ acolhimento demonstrado pelos profissionais influiu na avaliação dos usuários acerca da qualidade do atendimento, observando-se uma atitude de preservação da figura do médico. O nível de escolaridade da mãe não apresentou diferença significativa em relação à melhora apresentada.

Conclusões: a metodologia de tratadores mostrou-se adequada para a identificação e análise crítica das principais questões envolvendo a avaliação da resolubilidade de serviços de saúde. As doenças respiratórias na infância são uma condição traçadora importante para avaliação da qualidade na atenção básica.

Palavras-chave Atenção básica à saúde, Avaliação de serviços de saúde, Métodos de avaliação 


\section{Introdução}

Até o final da década de 1990, a ênfase do Sistema Único de Saúde (SUS) foi dada à garantia da universalidade com ampliação da acessibilidade aos serviços, expansão da cobertura assistencial e descentralização da gestão para estados e municípios. ${ }^{1}$ Nesse contexto, as avaliações em saúde focaram primordialmente os indicadores epidemiológicos, produzindo análises comparativas entre coeficientes e índices de morbimortalidade bem como os indicadores quantitativos de oferta de assistência. Igualmente, vários trabalhos analisaram a evolução dos repasses financeiros, com enfoque no aumento do controle orçamentário frente a descentralização impulsionada pelo governo federal. ${ }^{2}$

A partir da virada do século, como conseqüência da expansão experimentada pelos serviços de atenção básica, a política nacional de saúde é premida a efetivar o princípio da integralidade, surgindo, então, a necessidade da melhoria da qualidade da assistência e da resolubilidade dos serviços de saúde. ${ }^{3}$ Tais necessidades tem desencadeado iniciativas de avaliação que apesar dos esforços dispendidos temse revelado uma tarefa mais espinhosa do que os estudos sobre acessibilidade. Essa dificuldade, em parte decorre da carência de informações que permitam analise das reais condições de oferta e utilização dos serviços de saúde bem como das distintas interpretações decorrente da variabilidade dos conceitos utilizados nas abordagens de avaliação. 4

Apesar dessas dificuldades, não se pode prescindir de parâmetros para identificar determinantes da resolubilidade, de qualidade da atenção e das relações existentes entre elas. Entre os obstáculos de natureza metodológica para se classificar e graduar essas variáveis encontram-se os registros incompletos de atendimento, a multiplicidade de atores e de intervenções que interagem no processo de atendimento, o caráter extremamente dinâmico da evolução das doenças e a interferência de fatores externos aos serviços de saúde, bem como o difícil controle do viés produzido pela observação direta. ${ }^{5}$

Portanto, a busca de estratégias avaliativas que produzam retratos desta realidade, representa uma contribuição relevante para o planejamento, gestão e avaliação da atenção à saúde. O conhecimento acerca da insuficiência das fontes de informação capazes de iluminar as múltiplas facetas de objetos com tamanha complexidade; a consciência das dificuldades enfrentadas pelas técnicas de coleta, no sentido de obter dados confiáveis; e a noção da fragilidade dos procedimentos disponíveis de análise para prover explicações às perguntas formuladas, têm levado à seleção e combinação de estratégias metodológicas, conformando desenhos minimamente capazes de promover aproximações aceitáveis do grau de resolubilidade obtida e dos fatores que influenciam o desempenho da atenção básica. ${ }^{6}$

A partir da segunda metade dos anos 60, buscando fortalecer o referencial metodológico da avaliação em saúde, diversos autores propuseram modelos teóricos com base na abordagem sistêmica, cabendo a Donabedian o esforço mais bem sucedido de síntese dessa abordagem, que é compreendida por três categorias de análise, a saber: Estrutura, Processo e Resultado. ${ }^{7}$ Por se tratar de uma abordagem simples e direta para a coleta e análise de dados, optou-se por utilizar o referencial sistêmico proposto por Donabedian, no presente estudo.

Por sua vez, as condições traçadoras propostas por Kessner, ${ }^{8}$ para avaliar a qualidade da atenção ambulatorial, permitem a análise dos componentes de forma horizontal, tendo como guia patologias que atendam aos seguintes critérios: impacto funcional, tecnologia de intervenção conhecida e disponível, facilidade de diagnóstico, taxa de prevalência relevante, clareza e disponibilidade de técnicas diferenciadas de prevenção, tratamento e reabilitação.

Apesar de uso inicialmente restrito, devido aos rigorosos critérios para sua utilização, é considerada uma das abordagens mais úteis para avaliação da atenção ambulatorial por permitir a análise horizontal e seqüencial do atendimento prestado, possibilitando a avaliação de serviços de saúde de forma holística. Nos Estados Unidos, a Joint Commission on Accreditation of Health Care Organizations tem aplicado o método em seus processos de auditoria, do mesmo modo que o Sistema Público de Seguro de Saúde o tem utilizado no desenvolvimento de estudos de custo-efetividade. ${ }^{9}$ Pesquisas utilizando essa metodologia têm sido desenvolvidas em vários continentes para aferir a efetividade de novas tecnologias de saúde e para o desenvolvimento de métodos voltados à melhoria da qualidade da assistência hospitalar, em países do terceiro mundo. ${ }^{10,11}$

O presente artigo tem, portanto, como objetivo, avaliar, mediante a combinação de tais estratégias metodológicas, a resolubilidade no atendimento prestado por unidades básicas de saúde em um Distrito de Saúde do município de São Paulo.

\section{Métodos}

O presente estudo foi realizado entre setembro de 1991 a maio de 1992, no Distrito de Saúde da Brasilândia, que se localiza na região norte do muni- 
cípio de São Paulo, Brasil, e que possuía uma população estimada, à época da coleta de dados, de 253.028 habitantes. Um percentual de $14,1 \%$ dessa população vivia com menos de quatro salários mínimos por mês e apenas $3,4 \%$ auferia renda mensal superior a trinta salários mínimos. Mais da metade dos moradores $(52,8 \%)$ residia em favelas e cortiços e a mortalidade proporcional dos menores de um ano era de $11,3 \%$, sendo as broncopneumonias a principal causa de óbito $(13,3 \%)$. As Unidades Básicas de Saúde (UBS) sob responsabilidade municipal eram em número de sete. 12

A população alvo do estudo foi composta pelas crianças menores de cinco anos, devido à elevada proporção de atendimentos e, consequentemente, maior oferta de serviços prestados a esse segmento da população.

A Doença Respiratória da Infância (DRI) foi a condição traçadora selecionada tendo em vista a elevada freqüência como motivo de procura de atendimento, corroborado pelo fato de existir critérios normativos explícitos para diagnóstico e tratamento deste grupo de doenças, 13,14 os quais foram utilizados como parâmetro de comparação.

O tamanho da amostra foi determinado admitindo-se um limite superior de $10 \%$ para o erro na comparação da proporção de eventos encontrados nos casos estudados, sob hipótese de variabilidade máxima, tendo o cálculo tomado por base a expressão:

$$
\frac{\mathrm{n}=\mathrm{Z}^{2} \cdot \alpha / 2 \cdot \mathrm{p} \cdot \mathrm{q}}{\Delta^{2}}
$$

Onde o $Z^{2} . \alpha / 2$ é o percentil $97,5 \%$ da distribuição normal $Z^{2} \cdot \alpha / 2=1,96$, sendo $p$ e $q$ supostamente iguais a 0,5 (máxima variabilidade) e $\Delta=0,10$, o que significa que, em cada UBS, o tamanho mínimo da amostra (n) deveria ser de 96 crianças. A coleta de dados ocorreu com base na semana típica compreendendo no mínimo cinco dias úteis de atendimento. 15

Para a análise das condições estruturais dos serviços foi utilizado um protocolo baseado na avaliação das condições de eficiência, que incluiu entrevistas com mãe ou responsável pela criança e observações das condições de estrutura dos serviços de saúde in locum. 16

Para a análise do processo foram transcritos dos prontuários os registros referentes à anamnese, exame físico, diagnósticos e condutas, tomando por base o estudo de Melendez, 17 tendo como guia da coleta de dados o estabelecido pelas normas da World Health Organization (WHO). 14 Os dados de exame físico registrados permitiram a construção de quatro grupos de acordo com a freqüência dos achados da investigação.
A avaliação do resultado foi efetuada mediante a realização de duas entrevistas com mães ou responsáveis pelas crianças, atendidas na semana típica. $\mathrm{Na}$ primeira entrevista, ocorrida na unidade de saúde antes da consulta médica, se obteve informações sobre as condições socioeconômicas da família, as queixas da criança, a gravidade atribuída pelo médico ao quadro, a expectativa do acompanhante com relação à evolução da doença e sobre a utilização prévia de outros serviços de saúde devido às queixas. Na segunda entrevista, realizada no domicílio da criança, entre 7 e 14 dias após a primeira entrevista, registrou-se a evolução percebida dos pais ou responsável com relação às queixas da criança, as complicações referidas, a utilização de serviços e automedicação devido ao problema de saúde durante o período, bem como a opinião a respeito da qualidade do atendimento na UBS.

Foi definido como indicador da resolubilidade do atendimento, a remissão da queixa principal presente no momento da primeira consulta na UBS.

As variáveis sócio-demográficas consideradas como possíveis condicionantes do nível de resolubilidade foram a idade da criança, a escolaridade da mãe, a renda familiar e o número de moradores no domicílio. Essas variáveis foram selecionadas por serem as condições de vulnerabilidade e risco socioambiental que poderiam influenciar os resultados esperados com as condutas terapêuticas preconizadas pela normatizaçao técnica.

Foi considerada como variável relativa ao estado pregresso de saúde da criança, a gravidade atribuída pela mãe e pelo médico ao quadro apresentado pela criança no momento da consulta na UBS. O relato de procura anterior por atendimento em unidade de saúde, bem como a automedicação devido ao quadro respiratório que motivou a consulta da criança, foram variáveis relacionadas com a utilização de serviços.

A qualidade objetiva da consulta foi aferida avaliando-se a coerência entre os dados do exame físico em relação ao protocolo e à natureza da queixa, com base no registro feito pelo médico no prontuário. A qualidade subjetiva foi aferida com base na avaliação do serviço como um todo e do atendimento prestado especificamente pelo médico, feita pela mãe durante a segunda entrevista.

As variáveis foram codificadas no programa Statistical Package Social Sciense (SPSS) sendo empregados como testes estatísticos o $\chi^{2}$ com $5 \%$ de grau de significância.

A presente pesquisa foi submetida e aprovada pelo Comitê de Ética da Faculdade de Saúde Pública da Universidade de São Paulo. 


\section{Resultados}

Conforme cálculo amostral apresentado na seção Métodos, o número mínimo de crianças deveria ser de 96, porém em cada Unidade Básica de Saúde a amostra foi composta de 103 a 134, num total de 768 crianças. Houve durante o processo de coleta apenas nove recusas em participar, distribuídas nas sete unidades estudadas.

Foram verificados percentuais crescentes de melhora do estado de saúde em função do aumento da idade, registrando-se menor percentual $(69,4 \%)$ entre os menores de um ano e o maior percentual $(80,5 \%)$ na faixa etária de três a cinco anos. Houve diferença estatisticamente significativa nas faixas de menores de um ano e de um a dois anos. (Tabela 1)

Quanto ao nível de escolaridade da mãe observou-se uma diferença significativa de com melhora em mães analfabetas e sem melhora nas mães nos acima de primário completo. Esta diferença poderia estar refletindo o grau de exigência e de expectativa maior do ultimo grupo. Com relação à renda familiar per capita, houve percentuais mais elevados de "sem melhora" nas faixas acima de um salário mínimo per capita enquanto a diferença significativa de melhora foi encontrado na faixa de
0,25 a um salário mínimo per capita. (Tabela 1). O maior porcentual de "com melhora" foi verificado em famílias com rendimento menor que 0,25 e um salários mínimos per capita. Não houve diferença significativa na evolução da queixa em relação ao número de residentes no domicílio. De acordo com a classificação de gravidade baseada na avaliação médica foi observada uma diferença estatisticamente significante de melhor evolução dos quadros considerados como moderados (83,4\% dos casos). Para os quadros leves foi encontrada uma diferença significativa de sem melhora (Tabela 2).

A comparação dos quatro grupos de crianças, conforme descrito na metodológica, mostrou que o exame de apenas um órgão apresenta diferença significativa de sem melhora (Tabela 2).

Os percentuais de evolução favorável informada se mostraram semelhantes para as crianças que utilizaram previamente outro serviço de saúde ou que foram medicadas por pessoas que não eram profissionais de saúde e para aquelas que não recorreram a estas práticas $(76 \%)$.

Tanto as mães que consideraram ter tido o atendimento boa qualidade $(79,9 \%)$ quanto aquelas que o julgaram deficiente $(20,1 \%)$, basearam suas respostas no nível de acolhimento proporcionado

Tabela 1

Evolução clínica da doença respiratória aguda segundo características sócio-demográficas da população atendida em Unidades Básicas de Saúde. Distrito da Brasilândia, Município de São Paulo, 1991.

\begin{tabular}{|c|c|c|c|c|c|c|c|}
\hline \multirow[t]{3}{*}{ Indicadores } & \multicolumn{4}{|c|}{ Evolução da queixa } & \multirow[t]{3}{*}{ Total } & \multirow[t]{3}{*}{$\chi^{2}$} & \multirow[t]{3}{*}{ Valor de $p$} \\
\hline & \multicolumn{2}{|c|}{ Sem melhora } & \multicolumn{2}{|c|}{ Com melhora } & & & \\
\hline & $\mathrm{n}$ & $\%$ & $\mathrm{n}$ & $\%$ & & & \\
\hline \multicolumn{8}{|l|}{ Faixa etária (anos) } \\
\hline$<1$ & 108 & 30,6 & 245 & 69,4 & 353 & 21,80 & $<0,0001$ \\
\hline 1 a 2 & 46 & 15,2 & 256 & 84,8 & 302 & 16,64 & $<0,0001$ \\
\hline 3 a 5 & 22 & 19,5 & 91 & 80,5 & 113 & 0,89 & 0,345 \\
\hline Total & 176 & 22,9 & 592 & 77,1 & 768 & & \\
\hline \multicolumn{8}{|l|}{ Escolaridade materna } \\
\hline Não lê & 6 & 12,2 & 43 & 87,8 & 49 & 3,37 & 0,066 \\
\hline Primário incompleto & 42 & 18,8 & 181 & 81,2 & 223 & 2,97 & 0,085 \\
\hline Primário completo & 88 & 23,2 & 291 & 76,8 & 379 & 0,04 & 0,844 \\
\hline Médio e superior & 40 & 34,2 & 77 & 65,8 & 117 & 9,93 & 0,0016 \\
\hline Total & 176 & 22,9 & 592 & 77,1 & 768 & & \\
\hline \multicolumn{8}{|l|}{ Renda familiar } \\
\hline$<0,25$ salário mínimo & 22 & 28,9 & 54 & 71,1 & 76 & 1,74 & 0,187 \\
\hline 0,25-1 salário mínimo & 81 & 17,8 & 375 & 82,2 & 456 & 16,88 & $<0,001$ \\
\hline 1 salário mínimo e + & 59 & 28,1 & 151 & 71,9 & 210 & 4,39 & 0,036 \\
\hline Ignorado & 14 & 53,8 & 12 & 46,2 & 26 & 14,57 & $<0,001$ \\
\hline Total & 176 & 22,9 & 592 & 77,1 & 768 & & \\
\hline
\end{tabular}


Tabela 2

Evolução da queixa da doença respiratória aguda segundo gravidade clínica e número de órgãos essenciais examinados (org. ex) ao exame físico em Unidades Básicas de Saúde. Distrito da Brasilândia, Município de São Paulo, 1991.

\begin{tabular}{|c|c|c|c|c|c|c|c|}
\hline \multirow[t]{3}{*}{ Indicadores } & \multicolumn{4}{|c|}{ Evolução da queixa } & \multirow[t]{3}{*}{ Total } & \multirow[t]{3}{*}{$\chi^{2}$} & \multirow[t]{3}{*}{ Valor de $p$} \\
\hline & \multicolumn{2}{|c|}{ Sem melhora } & \multicolumn{2}{|c|}{ Com melhora } & & & \\
\hline & $\mathrm{n}$ & $\%$ & $\mathrm{n}$ & $\%$ & & & \\
\hline \multicolumn{8}{|c|}{ Gravidade clínica } \\
\hline Leve & 96 & 25,5 & 280 & 74,5 & 376 & 4,96 & 0,026 \\
\hline Moderada & 33 & 16,6 & 166 & 83,4 & 199 & 6,07 & 0,014 \\
\hline Grave & 8 & 25,0 & 24 & 75,0 & 32 & 0,11 & 0,735 \\
\hline Total & 137 & 22,6 & 470 & 77,4 & 607 & & \\
\hline \multicolumn{8}{|c|}{ Número de órgãos essenciais } \\
\hline 0 & 5 & 38,5 & 8 & 61,5 & 13 & 0,97 & 0,324 \\
\hline 1 & 17 & 39,5 & 26 & 60,5 & 43 & 6,85 & 0,009 \\
\hline 2 & 25 & 20,7 & 96 & 79,3 & 121 & 0,51 & 0,474 \\
\hline 3 & 131 & 22,2 & 460 & 77,8 & 591 & 1,47 & 0,224 \\
\hline Total & 178 & 23,2 & 590 & 76,8 & 768 & & \\
\hline
\end{tabular}

Tabela 3

Evolução da queixa da doença respiratória aguda segundo a qualidade do atendimento referido pelo acompanhante da criança e as sete Unidades Básicas de Saúde. Distrito da Brasilândia, Município de São Paulo, 1991.

\begin{tabular}{|c|c|c|c|c|c|c|c|}
\hline \multirow[t]{3}{*}{ Indicadores } & \multicolumn{4}{|c|}{ Evolução da queixa } & \multirow[t]{3}{*}{ Total } & \multirow[t]{3}{*}{$\chi^{2}$} & \multirow[t]{3}{*}{ Valor de $p$} \\
\hline & \multicolumn{2}{|c|}{ Sem melhora } & \multicolumn{2}{|c|}{ Com melhora } & & & \\
\hline & $\mathrm{n}$ & $\%$ & $\mathrm{n}$ & $\%$ & & & \\
\hline \multicolumn{8}{|c|}{ Qualidade do atendimento } \\
\hline Ruim & 8 & 47,1 & 9 & 52,9 & 17 & 4,28 & 0,038 \\
\hline Regular & 37 & 30,6 & 84 & 69,4 & 121 & 4,42 & 0,035 \\
\hline Bom & 133 & 21,1 & 497 & 78,9 & 630 & 8,40 & 0,004 \\
\hline Total & 178 & 23,2 & 590 & 76,8 & 768 & & \\
\hline \multicolumn{8}{|c|}{ Unidade Básica de Saúde } \\
\hline 1 & 24 & 22,9 & 81 & 77,1 & 105 & 0,01 & 0,933 \\
\hline 2 & 26 & 19,4 & 108 & 80,6 & 134 & 1,30 & 0,254 \\
\hline 3 & 24 & 23,3 & 79 & 76,7 & 103 & 0,00 & 0,974 \\
\hline 4 & 27 & 25,2 & 80 & 74,8 & 107 & 0,30 & 0,586 \\
\hline 5 & 22 & 20,4 & 86 & 79,6 & 108 & 0,56 & 0,455 \\
\hline 6 & 28 & 26,7 & 77 & 73,3 & 105 & 0,83 & 0,361 \\
\hline 7 & 27 & 25,5 & 79 & 74,5 & 106 & 0,36 & 0,546 \\
\hline Total & 178 & 23,2 & 590 & 76,8 & 768 & & \\
\hline
\end{tabular}


pelas pessoas que integraram a cadeia de atendimento e no tempo de espera para realização da consulta. Os médicos obtiveram $91,5 \%$ de aprovação sendo considerado bom "o médico que examina", "conversa", "dá atenção", "é educado".

Verifica-se pelo acima exposto que há diferença entre a opinião das mães quanto ao atendimento prestado pela UBS em seu conjunto e a opinião atribuída especificamente ao desempenho do médico. Na comparação desses dados com o tipo de evolução dos casos, verificou-se melhora informada em $78,8 \%$ daqueles em que as mães consideraram o atendimento bom. Já dentre as que consideraram o atendimento ruim houve melhora em apenas 52,9\% dos casos, sendo essa diferença estatisticamente significante pelos testes de $\chi^{2}$. (Tabela 3 ).

A análise da possível inter-relação entre a resolubilidade e a estrutura dos serviços não se mostrou útil face à semelhança das condições apresentadas pelas UBS no que diz respeito aos recursos disponíveis e aos aspectos organizacionais presentes.

A Tabela 4 mostra uma estreita amplitude de variação dos percentuais de evolução da queixa principal com e sem melhora entre as diferentes UBS.

\section{Discussão}

As doenças respiratórias em crianças menores de cinco anos continuam a constituir um dos principais problemas de saúde e, portanto, causa de importante demanda às unidades básicas de saúde. Essa patologia representa a terceira causa mais importante de anos de vida perdidos por incapacidade em crianças, em 2000, na região das Américas, conforme Grisi et al. 18

O painel de monitoramento das condições de saúde no município de São Paulo mostra claramente que as doenças respiratórias continuam sendo primeiro diagnóstico da rede de atenção básica neste município nos últimos quatro anos. 19

A utilização de metodologias de avaliação como instrumento de gestão, que possibilite analisar o alcance e as dificuldades em atender, pela tecnologia disponível nos serviços de saúde, as necessidades de saúde da população tem sido um desafio para o aprimoramento dos sistemas de saúde em todo o mundo. ${ }^{19}$ Nesse sentido, chama a atenção que os casos considerados moderados tenham apresentado melhor evolução, provavelmente devido ao fato de apresentarem sintomatologia mais evidente. A evolução desfavorável dos quadros considerados "leves" levanta a possibilidade de que a conduta do serviço não esteja se mostrando sensível para intervir nestes quadros o que implicaria em oportunidades perdidas de interrupção da história natural e/ou de prevenção de complicações.

O presente artigo se propõe a analisar os resultados utilizando uma lógica distinta da habitual no sentido de apontar que a avaliação deve ser guiada por objetivos, discutir os resultados partindo da evolução que seria a diretriz finalistica de serviços de saúde, que busquem a solução de problemas de saúde e não apenas a prestação de serviços. Observou-se o cuidado de discutir todos os resultados apresentados, mas seguindo uma lógica distinta que defendida no processo de avaliação, como instrumento de gestão.

A opinião das mães sobre a qualidade influiu na evolução da doença respiratória. Essa relação tem sido estudada num contexto de análise em que variáveis de controle de causas, risco e danos são levados em conta na evolução de doenças comuns na infância.21 A diferença entre os percentuais de aprovação ao atendimento realizado pelo profissional médico e o atendimento recebido na UBS como um todo é um fenômeno evidenciado neste trabalho. A valorização, talvez excessiva, atribuída pela clientela ao profissional médico na atenção à saúde, tem sido relatada em distintos contextos, nos quais esse profissional aparece sempre preservado do desgaste inerente ao cotidiano do serviço. $22 \mathrm{Na}$ experiência dos autores essa preservação da figura do médico decorre essencialmente da forma autoritária e ao mesmo tempo dependente em que a relação médico-paciente é construída nos serviços de saúde, principalmente pelo fato de que o usuário nunca sabe quando necessitará do médico novamente e em que condições de fragilidade.

O grupo das doenças respiratórias tem sido utilizado na análise da percepção da doença e na avaliação da acurácia dos diagnósticos realizada nos serviços de saúde como um eixo norteador da mediação de morbidade e na inter-relação com as dimensões da saúde. 23 Os resultados apresentados no presente estudo mostram que apesar da fragilidade do registro nos prontuários médicos, a evolução da queixa principal apresentou diferença significativa de acordo com a qualidade do registro do exame físico dirigido aos órgãos essenciais. Nesse sentido, o estudo mostra que o prontuário médico confirma o seu potencial de importante fonte primária na avaliação da qualidade da prestação de serviços.

Das condições socioeconômicas estudadas, identifica-se que o nível de escolaridade da mãe não se mostrou diretamente relacionado à evolução da queixa. Por outro lado, as crianças de famílias com rendimento menores do que 0,5 salários mínimos per 
capita foram as que apresentaram melhor evolução. É possível que para esta parcela da população não haja um nível de expectativa e de crítica capaz de levar a uma definição mais exigente do que seria informado como uma evolução favorável do caso.

A baixa influência demonstrada no padrão evolutivo da doença, seja pela procura anterior de atendimento para o caso, seja pela administração de medicamentos antes da consulta realizada na UBS, merece melhor investigação. A pesquisa revelou que $48 \%$ desta medicalização não foi prescrita pelo médico, o que coloca em dúvida a adequação de grande parte dos tratamentos até então instituídos. Além disso, não sendo esta uma pesquisa sobre utilização de serviços, prescindiu de investigar a forma de acesso aos medicamentos prescritos e à sua ministração, o que implicaria em aferir doses, intervalos e período de ministração, entre outros aspectos.

\section{Referências}

1. Levcovitz E, Lima LD, Machado CV. Política de saúde nos anos 90: relações intergovernamentais e o papel das Normas Operacionais Básicas. Ciênc Saúde Coletiva. 2001; 6: 269-91.

2. Novaes HMD. Avaliação de programas, serviços e tecnologias em saúde. Rev Saúde Pública. 2000; 34: 547-9.

3. Mendes EV. Os grandes dilemas do SUS. Salvador: Casa da Qualidade; 2001. Tomo 1.

4. Hartz ZMA. Pesquisa em avaliação da atenção básica: a necessária complementação do monitoramento. Divulg Saúde Debate. 2000; 1: 29-35.

5. Dal Poz MR, Pierantoni CR, Varella TC. Produtividade e desempenho dos recursos humanos nos serviços de saúde. Brasília, DF: Organização Panamericana de Saúde; 1997.

6. Lima LA, Gonçalves S, Lovisi G, Pereira BB. Validação transcultural da Escala de Avaliação de Limitações no Comportamento Social - SBS-BR. Rev Psiquiatr Clín. 2003; 30: 126-38.

7. Hartz ZMA, Vieira-da-Silva LM, organizadores. Avaliação em saúde: dos modelos teóricos à prática na avaliação de programas e sistemas de saúde. Salvador: Ed. EDUFBA; 2005.

8. Kessner DM. Assessing health quality: the case for tracers. N Engl J Med. 1973; 288: 189-94.

9. Fonseca AS, Yanamaka NMA, Barison THAS, Luz SFL. Auditoria e o uso de indicadores assistenciais: uma relação mais que necessária para a gestão assistencial na atividade hospitalar. Mundo Saúde. 2005; 29: 161-9.

10. La Vecchia C, Levi F, Lucchini F, Lagiou P, Trichopoulos D, Negri E. Development of a aspect tracer to image the distribution of replicating. Cancer. 1998; 83: 2223-7.
A metodologia de traçadores, proposta para o estudo da qualidade da atenção básica, mostrou-se adequada na identificação e análise crítica das principais questões envolvendo a avaliação da resolubilidade em serviços de saúde. As estratégias de avaliação e melhoria da qualidade na atenção básica têm sido direcionadas principalmente para a avaliação da Estratégia de Saúde da Família como a principal diretriz política para alcançar o princípio da universalidade no Sistema Único de Saúde (SUS). Cabe realçar que nesta avaliação se preconiza o uso de traçadoras para aprofundar a compreensão e interpretação de dados referentes à interação realizada nos serviços e a necessidade de se conhecer as expectativas dos usuários frente à queixa principal. 24 Estes deverão se constituir desafios para futuras investigações no campo da avaliação da qualidade focada em resultados.

11. Kaizen. Framework for better health care in Israel: a suitable initiative. Strat Direction. 2003; 19: 11-3.

12. São Paulo (Capital). Secretaria da Saúde. Plano plurianual de saúde: 1989-93. São Paulo; 1989.

13. São Paulo (Estado) Secretaria de Saúde. Sistema Unificado e Descentralizado de Saúde. Projeto Doenças Respiratórias na Infância: manual de condutas. São Paulo; 1988.

14. WHO (World Health Organization). Respiratory infections in children: management at small hospitals. Geneve; 1986.

15. Tanaka OY, Melo C. Avaliação de programas de saúde do adolescente: um modo de fazer. São Paulo: EDUSP; 2004.

16. OPAS (Organização Pan-Americana da Saúde). Avaliação de serviços de atendimento ambulatorial de adolescentes: estimativa de complexidade e condições de eficiência. Washington, DC; 1995.

17. Melendez RT. Evaluación de la atención médica: ensayo sobre métodos utilizados en nuestro médio: informe preliminar. Tec Hosp. 1971; 18: 39-57.

18. Speroto G. Fundamentação teórica para um sistema de triagem. In: Grisi S, Okay Y, Sperotto G. Estratégia de Atenção Integrada as Doenças Prevalentes na Infância AIDPI. Washington, DC; 2002. p. 43-52.

19. São Paulo (Capital). Secretaria de Saúde. Painel de monitoramento: junho 2007. Disponível em: http://www.capital .sp.gov.br/secretarias/saude/informaçoesemsaude. [2007 out. 10].

20. Starfield B. Atenção primária: equilíbrio entre necessidades de saúde, serviços e tecnologia. Brasília, DF: UNESCO, Ministério da Saúde do Brasil; 2004. 
21. Lopes RM, Silva LMV, Hartz ZMA. Teste de uma metodologia para avaliar a organização, acesso e qualidade técnica do cuidado na atenção à diarréia na infância. Cad Saúde Pública. 2004; 2 (Supl 2): 283-97.

22. Sucupira ACSL. Relações médico-paciente nas instituições de saúde brasileiras [dissertação mestrado]. São Paulo: Faculdade de Medicina da Universidade de São Paulo; 1981

Recebido em 18 de outubro de 2007

Versão final apresentada em 7 de abril de 2008

Aprovado em 10 de abril de 2008
23. Theme Filha MM, Szwatcwald CL, Souza Jr PRB. Medidas de morbidade referida e interrelações com dimensões da saúde. Rev Saúde Pública. 2008; 42: 73-81.

24. Campos CEA. Estratégia de avaliação e melhoria contínua da qualidade no contexto de Atenção Primária em Saúde. Rev Bras Saúde Matern Infant. 2005; 5: 563-9. 